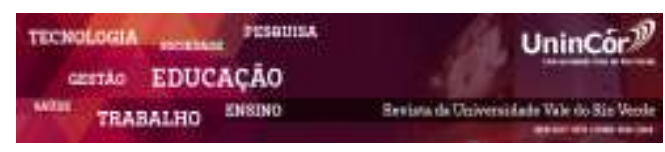

Revista da Universidade Vale do Rio Verde ISSN: 1517-0276 / EISSN: 2236-5362 v. $17 \mid$ n. 1 | Ano 2019

Andreia de Bem Machado Universidade Federal de Santa Catarina (UFSC) andreiadebem@gmail.com

Paulo Henrique da Silva

Delinea phs.paulohenriquesilva@gmail.com

\section{REVISÃO INTEGRATIVA: PLATAFORMAS DE REPOSITÓRIO DE CONTEÚDOS EDUCACIONAIS}

\begin{abstract}
RESUMO
A tecnologia trouxe inúmeros benefícios à sociedade moderna, revolucionando diversos setores da sociedade. Atualmente, ela vem mudando o formato na área da educação, propondo facilidades referentes a ferramentas de armazenamento nas nuvens. Sendo assim o objetivo desse artigo é : analisar por meio da revisão de literatura as plataformas de repositório de conteúdo educacionais. Para tanto, realizou-se a análise bibliométrica a partir de uma busca sistemática na base de dados Scopus. Como resultado, identificou-se que a pesquisa emerge no campo das Ciências Sociais, Ciência da Computação, Engenharia e Ciências da decisão.
\end{abstract}

Palavras-chave: Conteúdos educacionais. Repositório. Plataforma. Educação.

\section{INTEGRATIVE REVIEW: REPOSITORY PLATFORMS OF EDUCATIONAL CONTENT}

\begin{abstract}
Technology has brought countless benefits to modern society, revolutionizing various sectors of society. Currently, it has been changing the format in the area of education, proposing facilities related to cloud storage tools. Thus, the objective of this article is to: Analyze through the literature review the educational content repository platforms. To do so, the bibliometric analysis was performed from a systematic search in the Scopus database. As a result, it was identified that the research emerges in the field of Social Sciences, Computer Science, Engineering and Decision Sciences
\end{abstract}

Keywords: Educational contents. Repository. Platform. Education.

Recebido em: 03/12/2018 - Aprovado em: 31/03/2019 - Disponibilizado em: 15/07/2019

\section{INTRODUÇÂO}

Sociedade do conhecimento e da informação é a designação usada atualmente para intitular/designar o contexto social que em vivemos. Percebe-se que estamos vivenciando em uma nova fase de desenvolvimento histórico, ocorrida após o advento da internet que teve como consequência transformações atreladas principalmente a informação e a tecnologia. A tecnologia ganha espaço na sociedade servindo como mediação das ações concretizada no cotidiano que impulsiona, compartilha e dissemina a produção conhecimento e 
informação. Desde dos anos 2000, devido a essa relação entre tecnologia e informação, as discussões sobre autoarquivamento de informação, a repositórios, a colaboração e ao acesse livre vem sendo ampliados atingindo aos recursos didáticos educacionais.

Para Litto (2007), o início foi oriundo das interconexões de sistemas abertos (OSI), um conjunto de protocolos que garantiam que pessoas físicas e jurídicas, com máquinas e sistemas operacionais diferentes, pudessem comunicar-se entre si - interoperabilidade. Na sequência, o movimento Free and Open Source Software (Foss) concebeu o esforço de milhões de programadores espalhados pelo mundo, engajados na tarefa de produzir aplicações abertas para serem copiadas, modificadas e distribuídas de forma não comercial. O desenvolvimento e criação de "patentes abertas" (Patent Commons Project) e de revistas científicas on-line e abertas já faz parte dessa nova realidade. Porém o que provocou de modo mais direto todas as pessoas com desejo de aprender algo, formalmente ou informalmente, segundo o autor, é a abordagem designada Open Educational Resources - OER (Recursos Educacionais Abertos). Assim também se percebeu a necessidade da criação de plataformas que integrassem os conteúdos, mas além dessa integração com a nova modalidade de ensino educação a distância, houve também a preocupação com a produção de conteúdos educacionais para atender a essa oferta.

Com base nesta contextualização, o objetivo neste estudo é analisar por meio da revisão de literatura as plataformas de repositório de conteúdos educacionais.. Para tanto, o artigo está organizado em cinco seções. A primeira aqui explicitada, intitulada de introdução apresenta-se um contexto da pesquisa. Na segunda seção, descreve-se os procedimentos metodológicos trilhados na pesquisa. Na terceira seção, apresenta-se os dados, os resultados e uma análise bibliométrica desta pesquisa. Na quarta seção, faz-se as considerações finais apresentando a relevância das plataformas de repositórios digitais. Por último, na quinta seção, elencam-se as referências utilizadas.

\section{TRILHA METODOLÓGICA}

Para atender indagação desta pesquisa, trabalhou-se a partir de uma visão exploratóriadescritiva com o método indutivo com o objetivo de delinear o tema e ampliar a familiaridade dos pesquisadores com o fato a partir de dados suficientes permitindo ao pesquisador inferir uma verdade (MARCONI; LAKATOS, 2010).

Como método de pesquisa da literatura, utilizou-se a busca sistemática em uma base de dados on-line, seguida de uma análise bibliométrica dos resultados. A bibliometria é uma metodologia oriunda das ciências da informação que utiliza métodos matemáticos e estatísticos para mapear documentos e padrões de publicação (FEATHER; STURGES, 2003; SANTOS; KOBASCHI, 2009).

A bibliometria possibilita, a organização e análise quantitativa de dados relevantes como: produção por região; temporalidade das publicações; pesquisas por área do conhecimento; contagem da citação do estudo; fator de impacto de uma publicação científica entre outros. Esta análise permite a 
sistematização dos resultados de uma pesquisa e a minimização da ocorrência de possíveis vieses ao se olhar para uma determinada temática.

\subsection{Procedimentos Para Coleta De Dados}

Para a análise bibliométrica, o estudo foi organizado em três etapas distintas: planejamento, coleta e resultado. Estas etapas aconteceram de modo integrado para responder à pergunta norteadora da pesquisa: Quais plataformas são utilizadas para repositório de conteúdos educacionais? O planejamento iniciou-se no mês de agosto de 2018, quando a pesquisa foi realizada. No escopo do planejamento, foi definida como relevante a base de dados Scopus <http://www.scopus.com>, devido sua contribuição no meio acadêmico, seu caráter interdisciplinar, sua atualização constante e também por esta ser uma das maiores bases de resumos e referências bibliográficas de literatura científica revisada por pares.

Considerando-se que o problema de pesquisa, delimitou-se, na fase de planejamento, os termos de busca, a saber: "platform" and "repository for Content production for education". Como princípio básico para a busca, optou-se pela utilização dos termos nos campos "title", "abstract" e "keyword", sem restrição temporal, de idioma ou outra qualquer que possa limitar o resultado.

Com base no planejamento da pesquisa, a coleta de dados recuperou um total de 10 trabalhos indexados, com o primeiro registro datado de 2009 e o último de 2016.

Como resultado desta coleta de dados, identificou-se que os trabalhos foram escritos por 28 autores, vinculados a 16 instituições. Foram utilizadas 136 palavras-chave para identificar e indexar as publicações, que se apresentam distribuídas em 4 áreas do conhecimento. Identificou-se que do universo de 11 trabalhos científicos, 7 são conferências em papel e 4 artigos revisados por pares compondo a amostra para uma análise bibliométrica na área de Ciências Sociais; Ciência da Computação; Engenharia e Ciências da decisão o que permite tecer o estado da arte do tema a partir da base de dados consultada. $\mathrm{O}$ quadro 1 apresenta $\mathrm{o}$ resultado da coleta de dados numa análise bibliométrica geral dos resultados obtidos na base de dados Scopus.

Quadro 1 - Dados bibliométricos gerais obtidos na base de dados Scopus

\begin{tabular}{|c|c|}
\hline Base de dados & Scopus \\
\hline le busca & "platform " and "repository for Content production for education” \\
\hline Campos de busca & "title”, “abstract”, "keyword" \\
\hline $\begin{array}{c}\text { Total de trabalhos } \\
\text { recuperados }\end{array}$ & 10 \\
\hline Autores & 28 \\
\hline Instituições & 16 \\
\hline Países & 12 \\
\hline Palavras-chave & 136 \\
\hline Áreas do conhecimento & 4 \\
\hline Tipos de publicação & 2 \\
\hline
\end{tabular}

Fonte: Elaborado pelos autores (2018). 
Para a apreciação bibliométrica dos resultados fez-se a exportação do resultado para um software de gerenciamento bibliográfico denominado EndNoteWeb (software baseado na Web) e de modo integrado trabalhou-se com a organização de uma planilha de dados. Assim, as informações relevantes foram classificadas de acordo com: distribuição temporal; principais autores, instituições e países em evidência; tipo de publicação na área; principais palavras-chave e trabalhos mais referenciados.

\section{APRESENTAÇÃO DE DADOS E DISCUSSÕES}

Inicialmente analisou-se a distribuição temporal dos trabalhos, o que permitiu identificar que a primeira publicação esta datada de 2009 com dois artigos, seguido do ano de 2010 com dois artigos publicados. Nos anos de 2011 e 2012 houve apenas uma publicação na área. Já no ano de 2013 não houve publicação. No ano de 2014, houve um aumento nas publicações com duas publicações na área. No ano de 2015 segue com uma publicação e no ano de 2016 também com uma publicação. Sendo que no ano de 2017 e até 31 de agosto de 2018 não houveram publicações nessa área. Esta frequência mostra a descontinuidade e carência de pesquisa na área. Para melhor visualização elaborou-se o gráfico 1.

Gráfico 1 - Distribuição dos trabalhos por ano

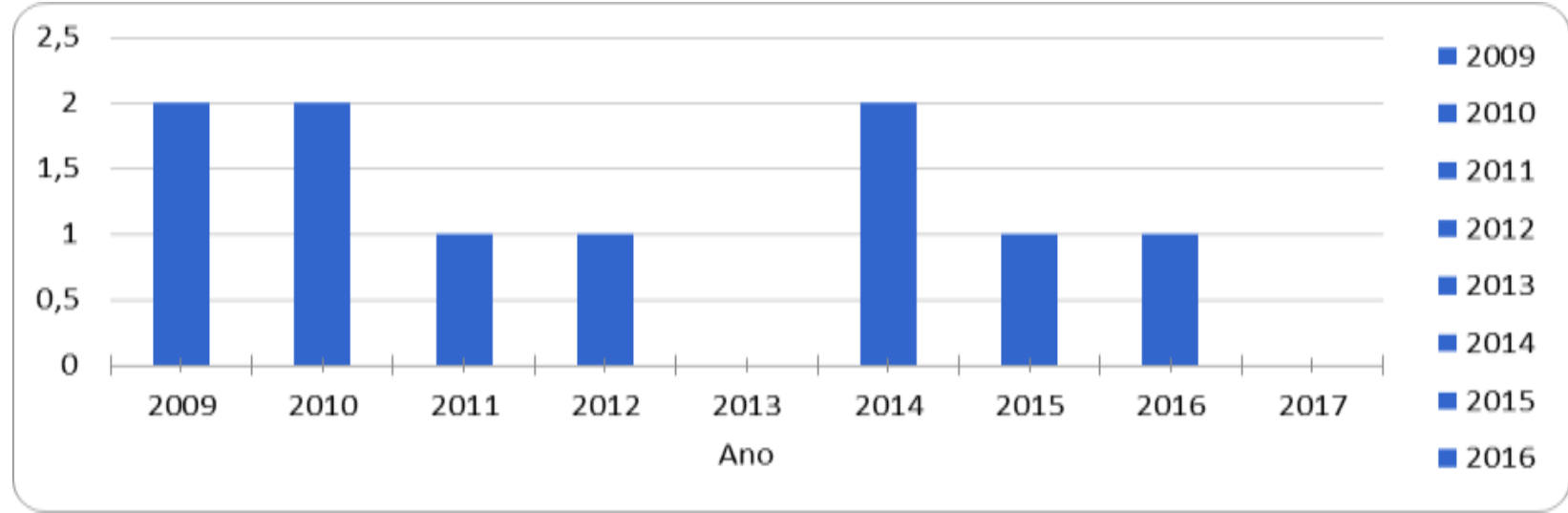

Fonte: Elaborado pelos autores (2018).

O primeiro artigo publicado em 2009 foi intitulado Open textbook proof-of-concept via connexions, nesse artigo de autores de Baker et al. (2009) foi discutido sobre o uso do Connexions como o repositório comum para conteúdo de livro aberto. Esse repositório
"Connexions" fornece uma plataforma de publicação para projetos de livros abertos. A outra publicação foi uma conferência publicado no mesmo ano intitulado Learning content production with XML and Java, de Alberto Gonzalez Tellez, esse artigo discute sobre a 
produção de conteúdo XML e Java e também sobre a entrega de conteúdo de aprendizagem nas plataformas.
Constatou-se a discussão sobre a temática de repositórios nos anos de 2010, 2011, 2012, 2014, 2015 e 2016 conforme quadro esquemático apresentado a seguir:

Quadro 2 - Resumo esquemático

\begin{tabular}{|c|c|c|c|c|}
\hline Ano & Autores & $\begin{array}{c}\text { Tipo de } \\
\text { Publicação }\end{array}$ & Título em Inglês & Resumo \\
\hline 2010 & $\begin{array}{l}\text { Alberto } \\
\text { González Téllez }\end{array}$ & Conferência & $\begin{array}{l}\text { Authoring environment for } \\
\text { e-learning production } \\
\text { based on independent } X M L \\
\text { formats }\end{array}$ & $\begin{array}{l}\text { Discute a criação do Docbook que é um } \\
\text { ambiente de autoria amigável, baseado } \\
\text { no produto independente, aberto } \\
\text { compatível com XML, utilizado na } \\
\text { educação a distância. }\end{array}$ \\
\hline 2010 & $\begin{array}{l}\text { Samanta Cueva } \\
\text { Carrión; } \\
\text { Germania } \\
\text { Rodríguez } \\
\text { Morales; } \\
\text { Audrey Audrey } \\
\text { Romero Peláez }\end{array}$ & Conferência & $\begin{array}{l}\text { OER'S production cycle } \\
\text { with social authorship and } \\
\text { semantic tools }\end{array}$ & $\begin{array}{l}\text { No artigo é discutido Recursos } \\
\text { Educacionais Abertos (OER) são } \\
\text { conteúdos digitais que são acessivis } \\
\text { através de repositórios da web. Eles são } \\
\text { usados como ferramentas de apoio ao } \\
\text { ensino superior. }\end{array}$ \\
\hline 2011 & $\begin{array}{l}\text { António Vieira } \\
\text { De Castro; } \\
\text { Carlos Vaz De } \\
\text { Carvalho; } \\
\text { Eurico Manuel } \\
\text { E.M. } \\
\text { Carrapatoso }\end{array}$ & Conferência & $\begin{array}{lrr}\text { Building a medical } \\
\text { learning } & \text { methodology } \\
\text { based on open } & \text { source } \\
\text { technologies } & \end{array}$ & $\begin{array}{l}\text { Discute a criação de repositório } \\
\text { temático denominado MELOR (Medical } \\
\text { Learning Objects Repository ) e uma } \\
\text { plataforma de e-learning denominada } \\
\text { MEDUCA que tem o objetivo de } \\
\text { investigar novos mecanismos para } \\
\text { aumentar a educação em saúde. }\end{array}$ \\
\hline 2012 & $\begin{array}{l}\text { Maria Eduarda } \\
\text { Rodrigues; } \\
\text { António } \\
\text { Moitinho } \\
\text { Rodrigues }\end{array}$ & Artigo & $\begin{array}{l}\text { Analyzing the performance } \\
\text { of an institutional scientific } \\
\text { repository - A case study }\end{array}$ & $\begin{array}{l}\text { O artigo analisa o desempenho do } \\
\text { RCIPCB (Repositório Científico do } \\
\text { Instituto Politécnico de Castelo Branco - } \\
\text { Portugal) considerando a evolução e } \\
\text { crescimento em termos de usuários, } \\
\text { arquivamento e auto-arquivamento, o } \\
\text { número de documentos publicados } \\
\text { (científicos) versus documentos } \\
\text { depositados em 2010 e } \\
\text { heterogeneidade entre comunidades } \\
\text { coleções e suas causas. }\end{array}$ \\
\hline 2014 & $\begin{array}{l}\text { Stefania } \\
\text { Biagioni; } \\
\text { Carlo Carlesi; } \\
\text { Joachim } \\
\text { Schöpfel; } \\
\text { Dominic } \\
\text { Farace; } \\
\text { Jerry Frantzen }\end{array}$ & Artigo & $\begin{array}{l}\text { Greyguide - guide to good } \\
\text { practice in grey literature: } \\
\text { A community driven open } \\
\text { resource project }\end{array}$ & $\begin{array}{l}\text { Aborda sobre a criação de um repositório } \\
\text { denominado GreyGuide que conterá } \\
\text { diretrizes para construçaõ de teses e } \\
\text { dissertações, como redigir relatórios de } \\
\text { pesquisa, metadados exigidos para } \\
\text { documentos de trabalho, bem como boas } \\
\text { práticas nas áreas de agricultura, saúde, } \\
\text { educação, energia, meio ambiente. }\end{array}$ \\
\hline 2014 & $\begin{array}{l}\text { Michael Organ; } \\
\text { Rebecca Daly }\end{array}$ & Artigo & $\begin{array}{l}\text { Embed and engage! } \\
\text { Delivering a digitisation } \\
\text { program at the University } \\
\text { of Wollongong Library }\end{array}$ & $\begin{array}{l}\text { É discutido no artigo um programa de } \\
\text { digitalização abrangente para suas } \\
\text { coleções exclusivas de arquivamento } \\
\text { pesquisa para Biblioteca da Universidade } \\
\text { de Wollongong. }\end{array}$ \\
\hline 2015 & $\begin{array}{l}\text { G. Isabel } \\
\text { Cristina Gomez; } \\
\text { T. Adalberto } \\
\text { Gabriel Diaz; } \\
\text { R. Claudia } \\
\text { Maria Zea; }\end{array}$ & Artigo & $\begin{array}{l}\text { Design of a competences } \\
\text { based teaching model } \\
\text { supported in the integration } \\
\text { of repositories and LMS } \\
\text { platforms for the automatic } \\
\text { control of processes course }\end{array}$ & $\begin{array}{l}\text { O artigo tem como objetivo discutir a } \\
\text { implementação de um conteúdo modelo } \\
\text { de gestão baseado em competências } \\
\text { suportando a integração de repositórios e } \\
\text { plataformas LMS. }\end{array}$ \\
\hline
\end{tabular}




\begin{tabular}{|c|c|c|c|c|}
\hline & $\begin{array}{l}\text { R. Luis Felipe } \\
\text { Zapata }\end{array}$ & & & \\
\hline 2016 & $\begin{array}{l}\text { Marangaze } \\
\text { Munhepe } \\
\text { Mulhanga; } \\
\text { Solange Rito } \\
\text { Lima; } \\
\text { Venâncio } \\
\text { Massingue }\end{array}$ & Conferência & $\begin{array}{l}\text { An evolutive model for } \\
\text { open science in } \\
\text { Mozambique }\end{array}$ & $\begin{array}{l}\mathrm{O} \text { artigo discute a implementação de } \\
\text { plataformas tecnológicas para a gestão } \\
\text { da ciência em Moçambique. }\end{array}$ \\
\hline
\end{tabular}

Fonte: Elaborado pelos autores (2018).

No cenário mundial temos a discussão de alguns repositórios entre eles: Docbook; ; MELOR;RCIPCB; GreyGuide. Também se constatou que há pesquisa sobre plataforma como a MEDUCA. Percebe-se que as pesquisas na área podem ser consideradas incipientes, pois apresentam-se, segundo análise quantitativa em pequena proporção e ascendência com momentos de descontinuidade, sendo que há um ano sem publicação.
A partir de um olhar sistêmico nas 10 publicações localizados, verifica-se uma variada lista de países que se destacam. Com destaque significativo para Portugal com três publicações, em segundo lugar França, Itália, Holanda e Espanha com duas publicações na área cada um dos países. O Brasil, segundo a pesquisa realizada não há publicações sobre essa temática indexada pela base de dados Scopus, conforme gráfico a seguir:

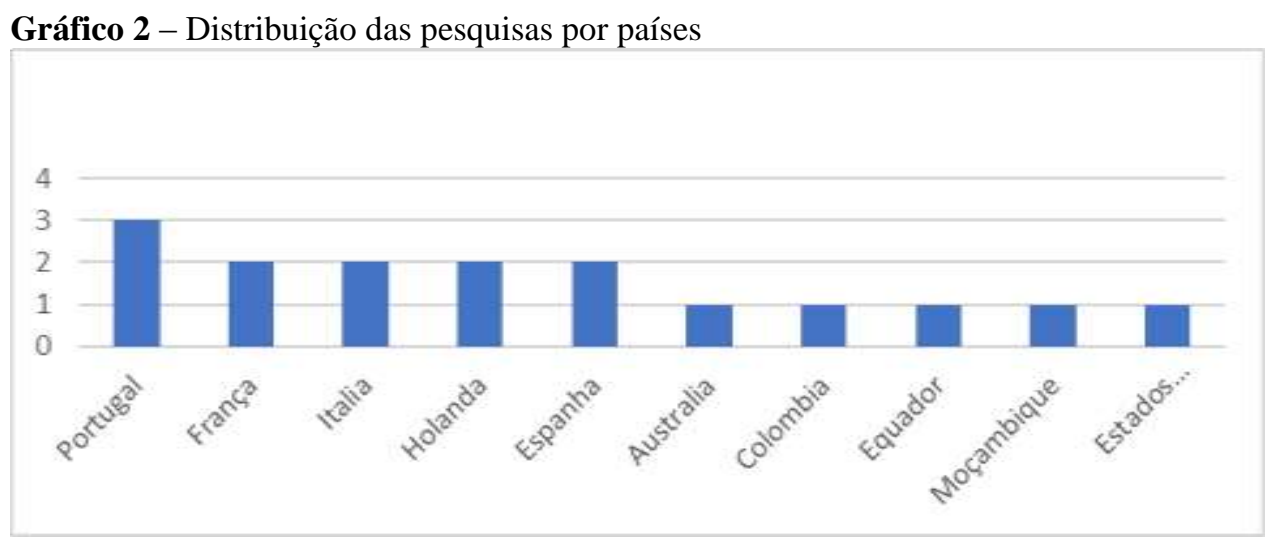

Fonte: Elaborado pelos autores (2018).

Com base no gráfico 2, a qual ilustra a frequência de publicações a respeito do tema em estudo, pode-se perceber que a pesquisa na área é relevante e que, de modo geral, ainda são incipientes as discussões sobre plataformas de repositório de conteúdos educacionais. Destacando o Brasil o país onde há mais discussões sobre essa temática. Assim dos 10 trabalhos filtrados na busca, destaca-se para essa pesquisa os autores que tem dois artigos publicados, conforme o quadro 2 a seguir: 
Quadro 2 - Autores com maior número de publicações na área, com suas afiliações e seus países.

\begin{tabular}{|c|c|c|c|}
\hline Autor & $\begin{array}{c}\text { Quantidade de } \\
\text { publicações }\end{array}$ & Afiliação & País \\
\hline $\begin{array}{c}\text { Stefania } \\
\text { Biagioni }\end{array}$ & 2 & Universidade de Pisa & Itália \\
\hline $\begin{array}{c}\text { Carlo Carlesi } \\
\text { Dominic } \\
\text { Farace }\end{array}$ & 2 & $\begin{array}{c}\text { Consiglio Nazionale delle } \\
\text { Ricerche }\end{array}$ & Itália \\
\hline $\begin{array}{c}\text { Jerry Frantzen } \\
\text { Joachim } \\
\text { Schöpfel }\end{array}$ & 2 & $\begin{array}{c}\text { GreyNet International } \\
\text { Universidade de Ciências } \\
\text { Aplicadas } \\
\text { Grupo de Estudos e } \\
\text { Pesquisa Interdisciplinar } \\
\text { em Informação e } \\
\text { Comunicação }\end{array}$ & Holanda \\
\hline
\end{tabular}

Fonte: Elaborado pelos autores (2018).

Por fim, buscando uma análise de cunho qualitativo percebeu-se que este debate envolve questões sobre plataformas de repositório de conteúdos educacionais, trazendo à tona questões como gerenciamento de produção de conteúdos e assim uma nova forma de fazer educação não se limitando a conteúdos científicos encontrados em livros e sim em recursos tecnológicos que proporcionam o acesso ao conhecimento. Permeando nestas discussões

\section{CONSIDERAÇÕES FINAIS}

A discussão sobre o tema plataformas de repositório de conteúdo educacionais ainda é incipiente. Há discussões em alguns países sobre plataformas e/ou repositórios já criados como é o caso dos repositórios: Docbook; ; MELOR;RCIPCB;GreyGuide e da plataforma MEDUCA. Percebe-se que esses foram criados a evolução das tecnologias de comunicação digital num processo amplo que envolve o aluno, professores, instituição de ensino, família e a sociedade. Contudo, não se identificou apesar da relevância e ênfase do tema plataformas de repositório de conteúdo educacionais na sociedade atual discussões permeando as metodologias e planejamento para elaboração dessas plataformas com intuito de utiliza-las na educação a distância.

com apenas uma utilidade que é de servir como repositório de conteúdo e/ou plataforma de informação e compartilhamento de uma determinada área do saber. Porém, há necessidade da criação de plataformas que integrassem os conteúdos, mas além dessa integração fazem a conexão com a modalidade de ensino educação a distância, havendo a preocupação com a produção de conteúdos educacionais para atender a essa 
oferta. Esse novo pensar educação exige-se em um novo formato na produção desse material didático, que requer gestão de projetos, para gerenciar a produção de material e também um banco de profissionais que possam executar e produzir para essa demanda.

O mapeamento científico da produção relacionada ao tema plataformas de repositório de conteúdo educacionais, permitiu uma análise bibliométrica que descrevesse as principais discussões da contemporaneidade e a interseção entre as áreas. Como resultado, identificou-se que a pesquisa emerge no campo multidisciplinar, intersectando as discussões nos campos: Ciências Sociais, Ciência da Computação, Engenharia e Ciências da decisão

Finalmente, evidenciou-se que as discussões sobre plataformas de repositório de conteúdo educacionais, no cenário mundial carecem de estudos e compartilhamento de boas plataformas que façam as integrações e interconexões entre recursos humanos, gestão de projetos e repositório educacional. Sugere-se, assim, que estudos nas diferentes áreas sejam na existência de um repositório de conteúdo que contenha a produção de conteúdo com visão macro (gestão de pessoas, conteúdos didáticos e gestão de projetos). Utilizando esse tripé para elaboração de material na modalidade educação a distância.

\section{REFERÊNCIAS}

ARAÚJO, Ulisses F. A quarta revolução educacional: a mudança de tempos, espaços e relações na escola a partir do uso de tecnologias e da inclusão social. Educação Temática Digital. v. 12, n 3, Campinas jan./abr. p. 31-48, 2011.
BAKER, Judy et al. Open textbook proof-ofconcept via connexions. International Review Of Research In Open And Distance Learning, Canadá, v. 5, n. 10, p.1-13, 01 nov. 2009. Mensal.

CYRINO, E. G., TORALLES-PEREIRA, M.L. Trabalhando com estratégias de ensinoaprendizado por descoberta na área da saúde: a problematização e a aprendizagem baseada em problemas. Cad. Saúde Pública, 2004; v. 20, n. 3), p. 780-788.

DEELMAN, Annechien; HOEBERIGS, Babet. A ABP no contexto da Universidade de Maastricht. In: ARAÚJO, Ulisses F. SASTRE, Genoveva (Orgs.). Aprendizagem baseada em problemas no ensino superior. $2^{\mathrm{a}}$ ed. São Paulo: Summus, 2009.

MARCONI, M. de A.; LAKATOS, E. M. Fundamentos de metodologia científica. São Paulo: Atlas, 2010.

SANTOS, R. N. M.; KOBASHI, N. Y.

Bibliometria, cientometria, infometria: conceitos e aplicações. Tendências da Pesquisa Brasileira em Ciência da Informação, Brasília, v. 2, n. 1, p. 155-172, 2009. Disponível em:

<http://inseer.ibict.br/ancib/index.php/tpbci/artic le/viewArticle/21>. Acesso em: 12 maio 2017.

TRENTIN, G. Telematics and on-line teacher training: the POLARIS Project. Journal Of Computer: Assisted Learning. Genova, p. 261270. 10 jan. 1997. Disponível em: <http://luigint.itd.cnr.it/Share. Acesso em: 12 maio 2017

\footnotetext{
Andreia de Bem Machado

Doutora no Programa de Pós-Graduação em Engenharia e Gestão do Conhecimento (PPEGC) na Universidade Federal de Santa Catarina (UFSC). Mestre em Educação Científica e Tecnológica (PPGECT) na Universidade Federal de Santa Catarina (UFSC). Especialista em Alfabetização na Universidade do Estado de Santa Catarina (UDESC). Graduada em Pedagogia na Faculdade de Educação (FAED) na Universidade do Estado de Santa Catarina (UDESC) Experiência na área de Educação há mais de 20 anos e com ênfase em Educação a Distância (EaD) desde de 2004, atuando principalmente nos temas: orientação de trabalho de pós-graduação a distância, planejamento, desenvolvimento, coordenação nos cursos a distância. Atualmente desenvolve trabalhos e pesquisas na área
} 
de planejamento, prática, acompanhamento e avaliação em EaD, educação corporativa, inovação, habitat de inovação, empreendedorismo, incubadora, parques tecnológicos bem como na área de Mídia e Gestão do Conhecimento. Atua também como professora formadora, palestrante, docente no ensino superior, professora orientadora e avaliadora de artigos científicos. Autora de livro e artigos

\section{Paulo Henrique da Silva}

Gestor de Projetos na Delinea. Especialização Área de estudo Qualidade e Engenharia de Software, Graduado em Ciência da Computação 